\title{
Cutinase Promotes Dry Esterification of Cotton Cellulose
}

\section{Zhao Xiaoman}

Key Laboratory of Eco-Textiles Ministry of Education, Jiangnan University, Wuxi 214122, P.R.China

Matama Teresa, Ribeiro Artur, and Silva Carla

Centre of Biological Engineering (CEB), University of Minho, Braga, Portugal

\section{Wu Jing}

State Key Laboratory of Food Science and Technology, Jiangnan University, Wuxi 214122, P.R.China

Fu Jiajia

Key Laboratory of Eco-Textiles Ministry of Education, Jiangnan University, Wuxi 214122, P.R.China

National Engineering Laboratory for Modern Silk, Soochow University, P.R.China

\section{Cavaco-Paulo Artur}

Centre of Biological Engineering (CEB), University of Minho, Braga, Portugal

International Joint Research Laboratory for Textile and Fiber Bioprocesses, Jiangnan University, Wuxi 214122, P.R.China

DOI 10.1002/btpr.2194

Published online November 28, 2015 in Wiley Online Library (wileyonlinelibrary.com)

Cutinase from Thermobifida fusca was used to esterify the hydroxyl groups of cellulose with the fatty acids from triolein. Cutinase and triolein were pre-adsorbed on cotton and the reaction proceeded in a dry state during $48 \mathrm{~h}$ at $35^{\circ} \mathrm{C}$. The cutinase-catalyzed esterification of the surface of cotton fabric resulted in the linkage of the oleate groups to the glycoside units of cotton cellulose. The superficial modification was confirmed by performing ATRFTIR on treated cotton samples and by MALDI-TOF analysis of the liquors from the treatment of the esterified cotton with a crude cellulase mixture. Modified cotton fabric also showed a significant increase of hydrophobicity. This work proposes a novel bio-based approach to obtain hydrophobic cotton. (C) 2015 American Institute of Chemical Engineers Biotechnol. Prog., 32:60-65, 2016

Keywords: biocatalysis, cellulose, cutinase, dry esterification

\section{Introduction}

Cutinase (E.C. 3.1.1.74) is a serine esterase whose natural substrate is cutin. Cutin, the structural component of the outer envelope (the cuticle) of higher plants, is a polyester. Cutinase is produced mainly by plant pathogens and it hydrolyzes the ester bonds of cutin thereby releasing fatty acids. Besides its natural substrate, cutinases are described to catalyze the hydrolysis of a variety of polymers, insoluble triacylglycerols, and low-molecular-weight soluble esters. ${ }^{1}$ In low water conditions, cutinases are also described as able to catalyze esterification and trans-esterification reactions. ${ }^{1}$ This versatile catalytic ability of cutinase is also described in a wide range of fiber modifications like wool anti-felting, ${ }^{2,3}$ cotton bio-scouring, ${ }^{4,5}$ and even synthetic fibers modification. $^{6-8}$ The enzymatic esterification of Avicel and cotton cellulose was reported ${ }^{9}$ using wild-type cutinase from Fusarium solani and an engineered cutinase, fused with the carbohydrate-binding module N1 from Cellolomonas fimi. The surface esterification of hemicellulose films catalyzed by lipases from Mucor javanicus, Rhizopus oryzae, Candida rugosa and by a cutinase from $F$. solani pisi is reported by

Correspondence concerning this article should be addressed to C.-P. Artur at artur@deb.uminho.pt. others. ${ }^{10}$ The specificities of cutinase and lipases were compared. Lipase presented higher activity toward long alkyl chain substrates while cutinase presented higher activity toward shorter alkyl chain substrates.

The interest in cellulose esters is evident from the diversity in type and applications of functionalized cellulose. ${ }^{11}$ Most developed processes involve homogeneous reactions where cellulose solubilization is a prerequisite. ${ }^{12}$ However, many industries, such as the paper and textile industries, have a particular interest in the hydrophobization of the cellulose surface rather than the extensive cellulose modification.

Hydrophobic surfaces are important because of the particular properties that they confer to a material such as water repellency, self-cleaning, friction reduction, and antifouling. ${ }^{13}$ From the textile industry perspective, surface modifications of cotton with long hydrophobic molecules would also improve the perception of softness, which can be considered to be an added-value. Traditional softeners are cation-active, anionic, nonionic surfactants that include waxes, paraffin, microemulsions, and other polymers. In the case of cotton, the cationic softeners ideally establish electrostatic bonds with cellulose fibers, so that the hydrophobic ends (which give soft handle) are directed outwards. ${ }^{14}$ Despite its hydrophilicity, cellulose has unmatched advantages as a substrate for the production of 
hydrophobic materials. These advantages include its abundance, biodegradability, and unique physical, chemical, and mechanical properties when compared to nonrenewable materials that are classically used. ${ }^{13}$ The modification of the surface of cellulose is accomplished in heterogeneous chemical or enymatic reactions, which are the subject of work aimed at developing greener alternatives that are either chemical- or enzymatic-based. ${ }^{15,16}$

The cutinases from $T$. fusca had shown a great potential for various textiles applications and were never reported before as usable for the esterification of carbohydrates. Here we report for the first time the direct modification of cotton in the dry state with triolein and cutinase from $T$. fusca aiming to modify the surface properties of the fabrics. The wettability of cotton was characterized by measuring the time of water drop adsorption. The linkage of the oleate groups to the glycoside units of cotton cellulose was verified by matrix-assisted laser desorption/ionization time-of-flight (MALDI-TOF) analysis of the liquors after cellulase treatment of treated cotton fabrics. Additional confirmation was provided by Fourier transform infrared (FTIR) analysis done directly on treated cotton samples.

\section{Materials and Methods}

\section{Reagents and enzymes}

Cotton fabric $\left(133 \times 72 / 40^{\mathrm{S}} \times 40^{\mathrm{S}}, 124 \mathrm{~g} / \mathrm{m}^{2}\right)$ was supplied by Zhejiang Buandong Dyeing and Garment (Zhejiang, China). The triolein (purity $\geq 98 \%$ ), the $n$-hexane (analytical grade) and the methyl alcohol (analytical grade) were purchased from Sinopharm Chemical Reagent (Shanghai, China). All the other chemical reagents were also purchased from Sinopharm Chemical Reagent (Shanghai, China). The cellulase (EC 3.2.1.4) from Trichoderma reesei with an activity of 6.0 units $/ \mathrm{mg}$ was obtained from Sigma-Aldrich Chemie GmbH (Steinheim, Germany). One cellulase unit is defined as the amount of enzyme that liberates $0.1 \mu$ mol of glucose from cellulose in $1 \mathrm{~h}$ at $\mathrm{pH} 5.0$ at $37^{\circ} \mathrm{C}$. The cutinase from $T$. fusca with an activity of $600 \mathrm{U} / \mathrm{mL}$ was kindly provided by the State Key Laboratory of Food Science and Technology, Jiangnan University (Wuxi, China). The activity of cutinase was determined by a continuous spectrophotometric assay utilizing p-nitrophenylbutyrate (pNPB) as the substrate. $^{2,17}$ One cutinase unit is defined as the amount of enzyme that liberates p-nitrophenol at the rate of $1 \mu \mathrm{mol} /$ min under the conditions of the assay.

\section{Pretreatment of cotton}

The cotton fabric samples with an average weight of $6.0 \mathrm{~g}$ were chemical bleached following the recipe described in literature. ${ }^{18}$ Briefly, samples were treated with $8.2 \%$ o.w.f. (of weight of fabric) of $\mathrm{Na}_{2} \mathrm{SiO}_{3} \cdot 9 \mathrm{H}_{2} \mathrm{O}, 1 \%$ o.w.f. of $\mathrm{Na}_{2} \mathrm{CO}_{3}, 3 \%$ o.w.f. of $\mathrm{NaOH}$, and $5 \%$ o.w.f. of $30 \% \mathrm{H}_{2} \mathrm{O}_{2}$, for $1 \mathrm{~h}$ at $90^{\circ} \mathrm{C}$. All the combined process of desizing, scouring, and bleaching were carried out in the L24A normal temperature oscillation machine (horizontal vibration, from Xiamen Rapid Precision Machinery, Xiamen, China) at a liquor ratio 20:1.

\section{Enzymatic modification of cotton fabric}

Bleached cotton samples were washed for $1 \mathrm{~h}$ with laundry detergent, rinsed thoroughly with water, dried at room

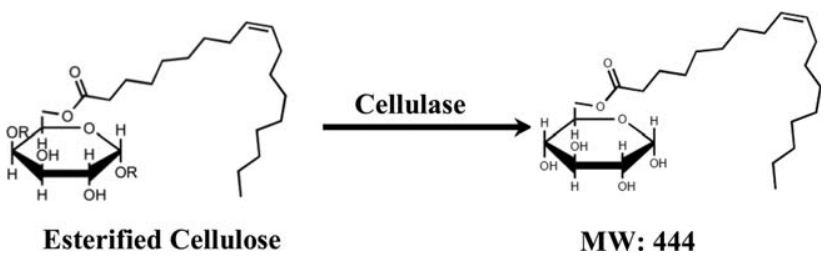

Figure 1. Schematic of the modified cellulose hydrolysis using cellulase.

Table 1. Relative Reducing Sugar Yield of Samples with Different Treatments

\begin{tabular}{lc}
\hline Cotton Fabric Samples & Normalized Amount of Reducing \\
Sugars (M)*
\end{tabular}

*All samples were measured in triplicate; the results have been normalized by subtracting the reference amount of reducing sugar in the liquid cellulase used for cotton hydrolysis; the values (mean $\pm \mathrm{SD}$ ) for cotton treated with active cutinase are significantly different from the values for cotton treated with inactive cutinase and the untreated cotton (one-way ANOVA; $p<0.0001$ ).

temperature, and then conditioned in the standard atmosphere for at least $48 \mathrm{~h}$ prior to the experiments. For all experiments, the enzymatic treatment of the cotton fabric substrates in Petri dishes was performed in the L-24A normal temperature oscillation machine (Shaoxing, China) operating at $35^{\circ} \mathrm{C}$ for $48 \mathrm{~h}$. Samples $(1000.0 \pm 0.1 \mathrm{mg})$ of cotton fabric were soaked with $2 \mathrm{~mL}$ of a solution containing cutinase $(600 \mathrm{U} / \mathrm{mL})$. Two millilitres of triolein were dripped into the samples after they were dried at room temperature. As controls, the cotton fabric was subjected to the same treatment but with heat-inactivated cutinase. Additionally, the untreated cotton fabric was used as the blank control.

After the enzymatic treatment, all cotton fabric samples were washed twice with $n$-hexane. ${ }^{9,19}$ All of the cotton samples were placed inside an extractor hood at room temperature for $48 \mathrm{~h}$ until solvent evaporation was complete.

\section{Cellulase hydrolysis of treated cotton}

Fabric samples treated with cutinase and blank controls were subsequently hydrolyzed by cellulase. The reactions were carried out in $1.5 \mathrm{~mL}$ of $0.2 \mathrm{M}$ sodium acetate - acetic acid buffer at $\mathrm{pH} 5.0$ and $0.5 \mathrm{~mL}$ of cellulase with an activity of 30 units $/ \mathrm{mL}$ at $50^{\circ} \mathrm{C}$ for $24 \mathrm{~h}$. The blank control was the untreated cotton hydrolyzed by cellulase under the same conditions. Experiments were run in duplicate. The liquors of treatment were centrifuged and the supernatants (liquid samples) were analyzed for reducing sugars (see methods below) and by MALDI-TOF (see methods below).

\section{Water drop test}

The wettability of cotton samples was measured by the droplet test where the time in seconds for a drop of water to sink into the fabric is recorded. All the samples were conditioned in the standard atmosphere at room temperature $\left(20^{\circ} \mathrm{C}\right)$ and $60 \%$ of relative humidity for at least $48 \mathrm{~h}$ prior to the test. The process of the water-drop penetrating into samples was recorded by a fast recording video camera from Data Physics Corporation, German. The volume of each 
drop of liquid was $5 \mu \mathrm{L}$. Every injection was performed by the syringe of the optical contact angle measuring instrument to ensure each testing under the same conditions. The time when the water drop started to fall down on the sample surface and the time when the fallen water drop penetrated completely into samples were recorded, respectively. Then the differential between the two time points represented the total time that one water drop completely penetrated into the fabric sample. Measurements were made in five different points for each sample. Minor amounts of Bromphenol blue dye was dissolved in water used to better visualize the differences on the surface of cotton samples.

\section{FTIR spectroscopy}

Spectra of cotton fabric controls and samples after cutinase treatment were recorded using a Nicolet iS10 FT-IR spectrometer (Thermo Fisher Scientific, Shanghai, China) with a Golden Gate ATR accessory with a single reflection monolithic diamond. All the absorbance spectra were acquired in the range of $4000-400 \mathrm{~cm}^{-1}$ with a $4 \mathrm{~cm}^{-1}$ spectral resolution. Three different measurements were performed per sample.

\section{Reducing sugars}

The reducing sugars were measured by dinitrosalicylic (DNS) colorimetric method on BioTek's Synergy ${ }^{\mathrm{TM}} \mathrm{Mx}$ Microplate Reader and Spectrometer (USA) at $540 \mathrm{~nm}$ of wavelength. Regarding the preparation of dinitrosalicylic acid (DNS) solution, different ingredients used for the preparation are as follows: distilled water of $1416 \mathrm{~mL}, 3,5$-Dinitrosalicylic acid of $10.6 \mathrm{~g}$, and $\mathrm{NaOH}$ of $19.5 \mathrm{~g}$ were all dissolved gently in water

Table 2. Wettability of Cotton Fabric Samples Treated with Active Cutinase and Heat-Inactivated Cutinase

\begin{tabular}{lc}
\hline Cotton fabric samples & Absorption time/s* \\
\hline Untreated cotton & $<0.01$ \\
Treated with active cutinase & $1.91 \pm 0.09$ \\
Treated with inactive cutinase & $0.57 \pm 0.03$
\end{tabular}

*The values (mean $\pm \mathrm{SD}$ ) result from five measurements, the difference in absorption time for the cotton treated with active cutinase was statistically significant compared to the values for cotton treated with inactive cutinase and the untreated cotton (two-sample $t$-test; $p<0.0001$ ). bath at $50^{\circ} \mathrm{C}$ until a clear solution was obtained. Then the following chemicals were added: Rochelle salt of $300 \mathrm{~g}$, Phenol of $7.5 \mathrm{~mL}$ and sodium meta bisulphate $8.3 \mathrm{~g}$. After dissolving all the above ingredients, the solution was stored at room temperature in an amber colored bottle to avoid photo oxidation. ${ }^{20}$ About $200 \mu \mathrm{L}$ of per sample and $150 \mu \mathrm{L}$ of DNS solution were incubated in boiling water for $5 \mathrm{~min}$, then diluted by $2.15 \mathrm{~mL}$ of distilled water after cooling down using running cold water. And $200 \mu \mathrm{L}$ of prepared sample were tested at $540 \mathrm{~nm} .^{21,22}$

\section{Maldi-TOF}

The mass spectra of the products were acquired on a ultrafleXtreme MALDI-TOF/TOF mass spectrometry (Bruker Daltonics $\mathrm{GmbH}$, Germany) equipped with a $337 \mathrm{~nm}$ nitrogen laser. The matrix- 2,5-dihydroxy-benzoic acid (DHB) of $20 \mathrm{mg} / \mathrm{mL}$ was prepared in a solution of $10 \% \mathrm{EthOH}$ and $1 \mathrm{mM} \mathrm{NaCl}$ solution, and then mixed with samples (1:1). A volume of $2 \mu \mathrm{L}$ of each mixture sample were spotted onto a ground steel target plate (Bruker part $n^{\circ} 209519$ ) and analyzed by the method of RP700-3500 in the reflective positive mode.

\section{Results and Discussion}

The esterification of cellulose by oleate (18C) on glycoside units of cellulose was performed at the surface of cotton fiber. Since enzymes will not penetrate the bulk of fiber, ${ }^{9}$ it is expect that only the glycosidic units at the surface will be modified. To confirm the interfacial modification, we used cellulase enzymes to hydrolyze the surface of the treated cotton. As expected, we found oligomers linked the oleate groups (see Figure 1 and discussion below). Each cellulose oligomer chain contains at its end a reducing glycosidic unit, which it is known as a reducing sugar. The different levels of reducing sugar in the liquids after $24 \mathrm{~h}$ of hydrolysis with a commercial cellulose are shown in Table 1. As expected, a slightly lower level of reducing sugars was found in the case of cotton presumed to be functionalized.

The presence of reducing sugars in the supernatant of samples of all the cotton samples give the proof that the surface of cotton was hydrolyzed by cellulase enzymes. The results have been normalized by subtracting the reference amount of reducing sugar in the liquid cellulase used for cotton hydrolysis.

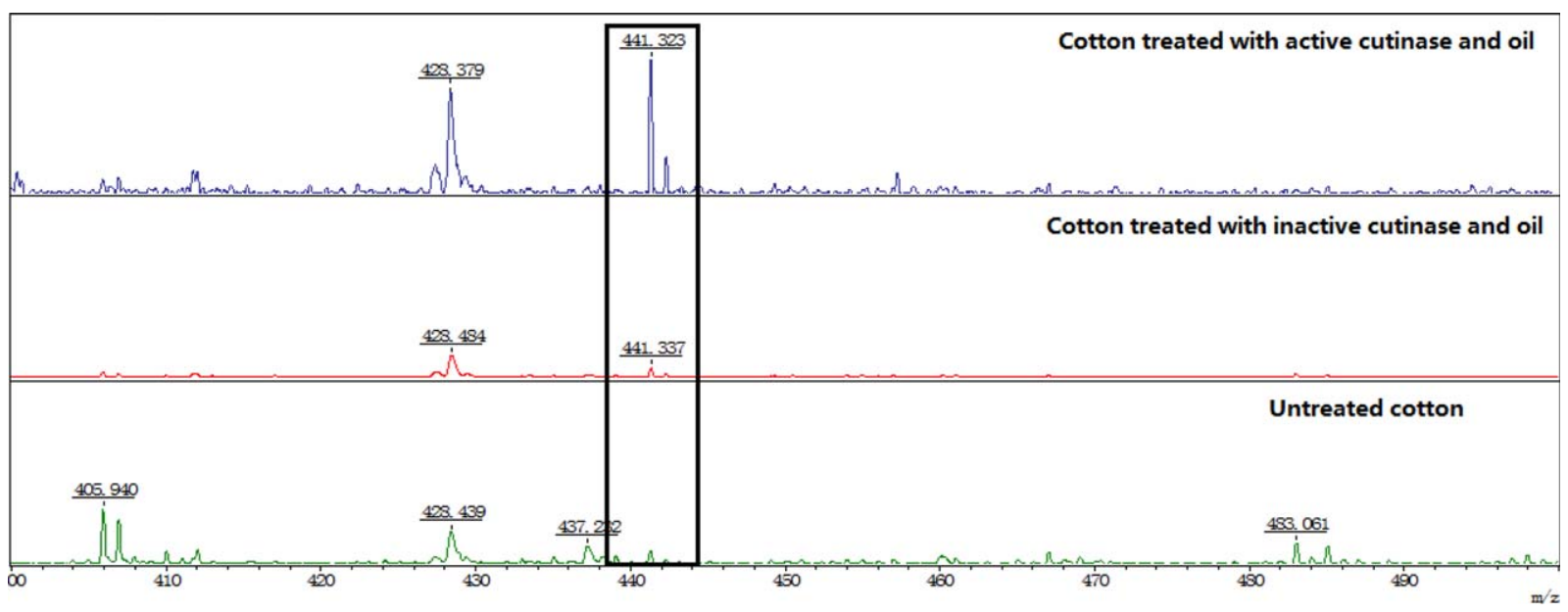

Figure 2. MALDI-TOF mass spectra of the hydrolysis solution of cotton treated with active cutinase, cotton treated with inactive cutinase and untreated cotton. 


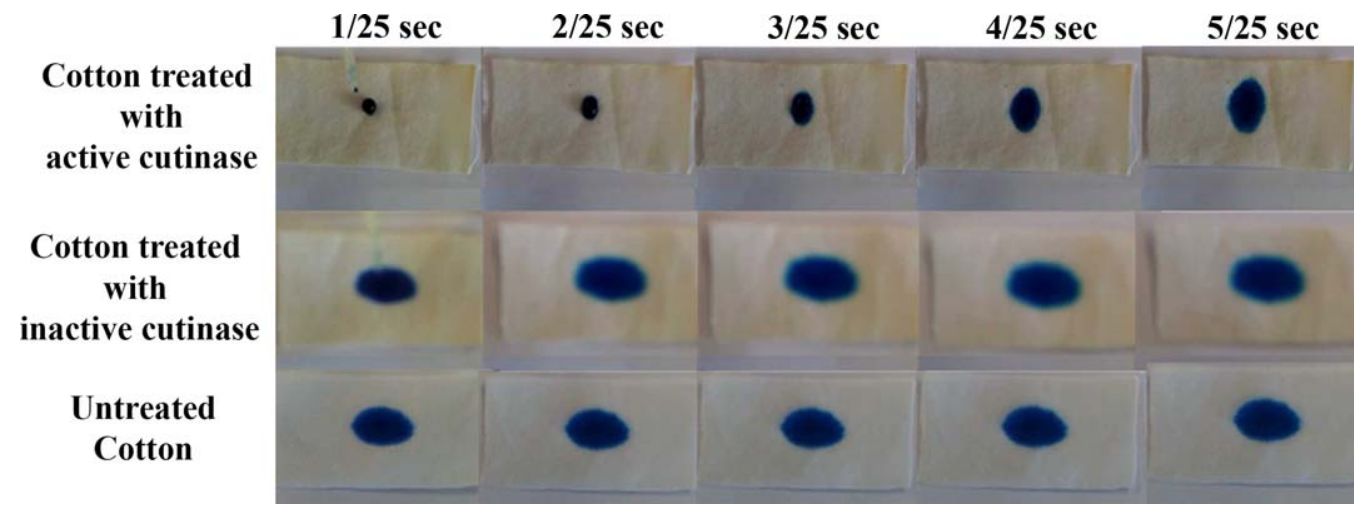

Figure 3. Comparison of the absorption profiles of a droplet of bromophenol blue solution in cotton sample treated with active cutinase, cotton sample treated with inactive cutinase and the untreated cotton sample.

The cotton samples treated with the inactive cutinase show a higher level of reducing sugars (Table 1) and slightly higher hydrophobicity (Table 2). These results seem to indicate that the wash-off with hexane of the inactive cutinase was not complete. Cutinase is known to be a hydrophobic protein $^{1}$ which could explain the high hydrophobicity. Once could speculate that the adsorbed cutinase could promote staining of cellulases enzymes which are highly glycosylated enzymes and therefore the increase of reducing sugar. The explanation of such results is not completely clear to us.

\section{MALDI-TOF mass analysis}

Figure 1 represents the scheme of cellulase action on modified cotton fabric. The cellulose oligomers formed by cellulase hydrolysis of unmodified and modified cotton samples were analyzed by MALDI-TOF to identify glucose units from the cellulose fibers covalently linked with oleic acid. The liquid containing cellulase enzymes was left under acetate buffer at room temperature for a few days. Therefore all cellulose oligomers were hydrolyzed to glucose and they were not found on MALDI spectra of the liquid of treatment. Residual amounts of glucose linked with oleate found indicate craft reaction. Via cutinase catalysis, oleic acid possibly successfully grafted to an unknown carbon position of the glycoside units of cellulose using triolein as substrate. The esterified product at C6 is represented; this position is in theory the most accessible for the enzyme.

The MALDI-TOF mass spectra results are present in Figure 2.

As shown in Figure 2, the mass of glucose covalently linked with the oleic acid was observed at $441 \mathrm{~m} / \mathrm{z}$, even though the intensity was weak. The slight difference between the molecule weight (MW) of modified glucose and the MW shown in the spectra was because of the probable loss of protons during the ionization process. For the cotton treated with inactive cutinase and untreated cotton samples, the target peak corresponding to the modified glucose was residual with the cotton treated with active cutinase. The detection in the MALDI-TOF spectrum of the glucose covalently linked to oleic acid supports the modification of the surface of cotton fabric by cutinase and triolein.

\section{Cotton wettability}

In order to have a qualitative determination of the acylation of cellulose surface with oleic acid by the cutinasecatalyzed esterification using triolein as substrate, the wett-

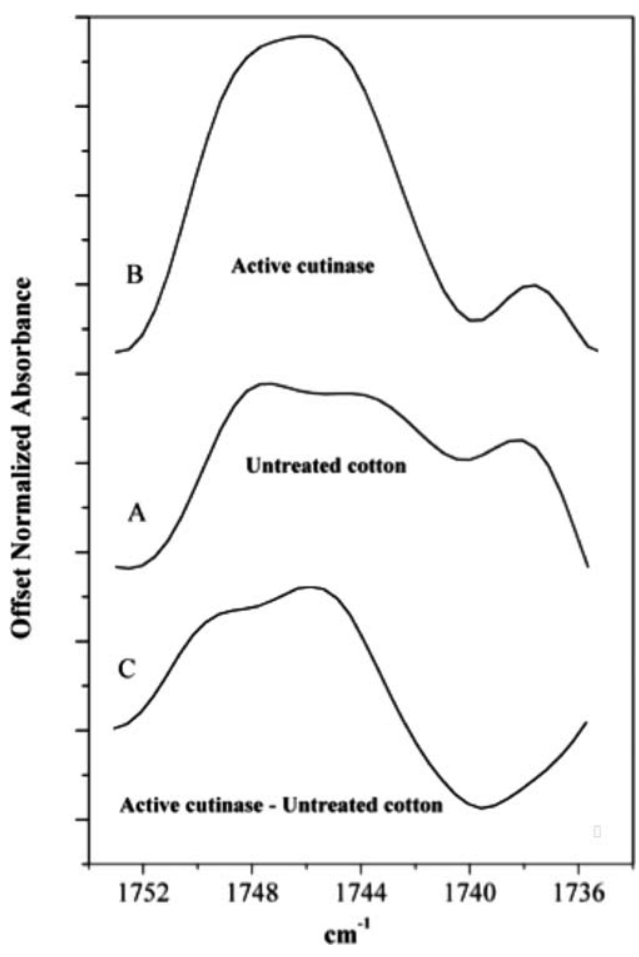

Figure 4. FTIR spectra of the untreated cotton and cotton samples treated with active cutinase.

ability of cotton samples was assessed by measuring the time that one water drop took to be completely absorbed after contact with the surface of cotton samples. As surface wettability is related with the hydrophobicity/hydrophilicity of the surface, the shorter the absorption time interval, the better the wettability of the cotton sample. The cotton samples' surface wettability are shown in Table 2.

As expected from a successful cellulose acylation, more time was necessary for one drop of water to penetrate into the cotton samples treated with active cutinase than the time needed in samples treated with inactive cutinase (control). The absorption time in the cotton samples treated with active cutinase was nearly three times longer than the control sample. The presence of chemically bonded long acyl chains to the cotton surface after active cutinase treatment and after proper hexane wash, has contributed to the hydrophobization of the cotton samples.

The heterogeneous esterification of macroscopic and microscopic cellulose using enzymes, mainly cutinase and lipases, is 


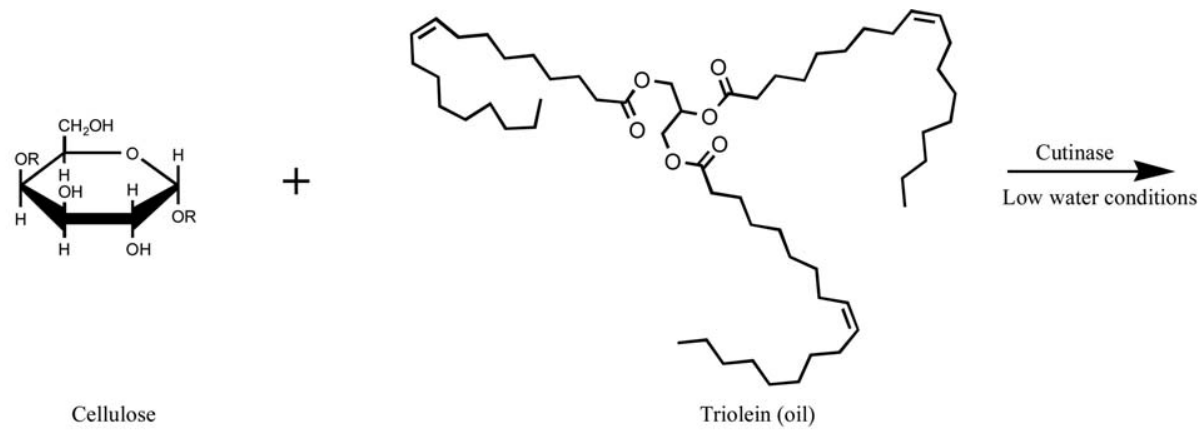

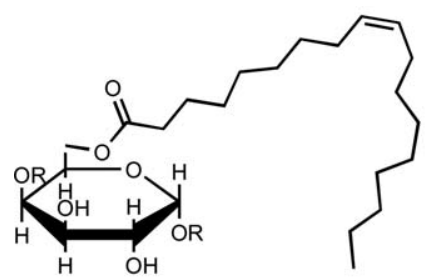

Modified cellulose Esterified cellulose

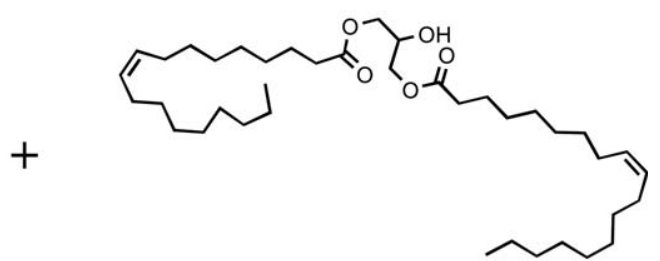

By-product

Figure 5. Cutinase-catalyzed esterification of triolein to the surface of cotton fabric.

reported by few groups. ${ }^{19,23}$ Only plain woven cotton was used as substrate for cutinase-mediated esterification by Matama et al. ${ }^{9}$ In this work we present a new approach for the simple and direct biomodification of hydroxyl groups on the surface of cotton fabric cellulose - a solvent-free process, using cutinase from $T$. fusca, which presents a higher thermal stability than cutinase from $F$. solani. Using the methodology here reported and using an important control-inactivated cutinase, we demonstrated that the time to completely absorb a drop of water on the cutinase-treated cotton fabric was significantly increased compared to the control. Surface wettability is a very complex physical phenomenon. ${ }^{24}$ It was not possible to compare the reduction of the wettability of cotton fabric reported by us with other wettability reductions because of the difference of the materials used. Nevertheless, the longest absorption time was below $2 \mathrm{~s}$, meaning that the bio modification of cotton fabric was not enough to reduce cotton wettability to levels that will affect its wearing comfort. Using a water solution containing bromophenol blue, it was possible to gather more evidence for the water-repellent effect that arises from the modification of cotton by cutinase (Figure 3). The time that the bromphenol blue droplet took to be absorbed further supports the hydrophobic modification of cotton already described.

\section{FTIR analysis}

FTIR was performed to further evaluate directly the esterification of triolein using cutinases on the surface of cotton samples. Spectral data were linearly normalized and the peaks were integrated in the range of 1735 and $1753 \mathrm{~cm}^{-1}$, typical for ester bond stretching vibration, using the OriginPro 8.5.0 SR1 software (OriginLab Coorporation, Northhampton, USA); no smoothing functions were used. Figure 4 shows the average individual spectra for the enzymatic treated cotton sample (Figure 4 curve B) and the original cotton (Figure 4 curve A) using triolein as the acid substrate. The most important absorption peaks at 1745 and $1748 \mathrm{~cm}^{-1}$ were assigned to the $\mathrm{C}=\mathrm{O}$ stretching and vibration, respectively, for cotton treated with active cutinase. The peak position depends on several factors like the posi- tion of the esterification carbon in the glycoside. ${ }^{9}$ The absorption peak at $1745 \mathrm{~cm}^{-1}$ for cotton treated with active cutinase was significantly stronger (56\% of increase in peak area) than that for the control sample. After subtracting the control spectrum, the resulting curve (Figure 4 curve C) showed a prominent peak at $1745 \mathrm{~cm}^{-1}$. These results independently confirm the MALDI-TOF mass spectra results on the modification of glucose. The FTIR additionally supported the esterification of cotton cellulose by cutinase using triolein as substrate. No significant changes were observed on other parts of FTIR spectra.

The results of MALDI-TOF mass spectroscopy and FTIR spectra were conclusive and demonstrated that cutinase catalyzed the esterification of oleic acid from triolein oil to the cotton surface, using a simple, solvent-free methodology, that can potentially be scaled up for use at an industrial scale.

Badenes et al. ${ }^{25}$ have shown the enzymatic transesterification between a mixture of triglycerides (oils) and methanol in AOT reversed micellar system, using recombinant cutinase from $F$. solani pisi as a catalyst. Theoretically, the ester group of oils can be transferred to alcoholic hydroxyl group of cellulose in cotton fabric especially with the catalytic ability of cutinase to use solid substrates. Accordingly, we demonstrated by FTIR and MALDI-TOF mass spectroscopy that the cutinase-catalyzed esterification between oils and the glycoside unit of cellulose was accomplished, resulting in the surface modification of cotton fabric. This process may have the potential to endow cotton fabrics excellent hydrophobic properties. Another positive outcome is the treated cotton samples are perceived to be softer. The model reaction of the cutinase-catalyzed esterification proposed for the enzymatic reaction is shown in Figure 5.

\section{Conclusions}

The cutinase-catalyzed transesterification between a mixture of oils and methanol for biodiesel production has been reported, ${ }^{26-28}$ but this type of enzyme reaction technology has not yet been applied in the textile industry. We previously reported the esterification of cellulose using cutinase, but here 
we report a novel solvent-free methodology, where the reactants are previously dried at the surface of the cellulose fabrics. The MALDI-TOF mass spectroscopy results proved the existence of glucose linked to oleic acid molecules. The characteristic groups of the target ester product on the surface of cotton fabric appeared at the characteristic infrared group frequencies in the FTIR spectra. Moreover, the hydrophobicity of the modified cotton fabric was significantly increased. This simple and straightforward approach has the potential to be scaled to the industrial level.

\section{Acknowledgments}

This work is supported by Opening Project of National Engineering Laboratory for Modern Silk (Grant No. KJS1312); the National Natural Science Foundation of China (Grant No. 31201134 and No. 31470509); the Jiangsu Provincial Natural Science Foundation of China (Grant No.BK2012112), the Industry-academic Joint Technological Prospective Fund Project of Jiangsu Province (Grant No.BY2013015-24) and the Graduate Funding Program of Jiangnan University for Overseas Study.

\section{Literature Cited}

1. Chen S, Su L, Chen J, Wu J. Cutinase: Characteristics, preparation, and application. Biotechnol Adv. 2013;31:1754-1767.

2. Wang P, Wang Q, Fan X, Cui L, Yuan J, Chen S, Wu J. Effects of cutinase on the enzymatic shrink-resist finishing of wool fabrics. Enzym Microb Technol. 2009;44:302-308.

3. Wang P, Yuan J, Ren X, Cui L, Wang Q, Fan X. Bio-antifelting of wool based on mild methanolic potassium hydroxide pretreatment. Eng Life Sci. 2013;13:102-108.

4. Yan H, Hua Z, Qian G, Wang M, Du G, Chen J. Effect of cutinase on the degradation of cotton seed coat in bio-scouring. Biotechnol Bioprocess Eng. 2009;14:354-360.

5. Zhang Y, Chen S, He M, Wu J, Chen J, Wang Q. Effects of Thermobifida fusca cutinase-carbohydrate-binding module fusion proteins on cotton bioscouring. Biotechnol Bioprocess Eng. 2011;16:645-653.

6. Ribitsch D, Acero EH, Greimel K, Eiteljoerg I, Trotscha E, Freddi G, Schwab H, Guebitz GM. Characterization of a new cutinase from Thermobifida alba for PET-surface hydrolysis. Biocatal Biotransfor. 2012;30:2-9.

7. Stavila E, Arsyi RZ, Petrovic DM, Loos K. Fusarium solani pisi cutinase-catalyzed synthesis of polyamides. Eur Polym J. 2013; 49:834-842.

8. Zhang Y, Wang L, Chen J, Wu J. Enhanced activity toward PET by site-directed mutagenesis of Thermobifida fusca cutinase-CBM fusion protein. Carbohydr Polym. 2013;97:124-129.

9. Matama T, Casal M, Cavaco-Paulo A. Direct enzymatic esterification of cotton and Avicel with wild-type and engineered cutinases. Cellulose 2013;20:409-416.

10. Stepan AM, Anasontzis GE, Matama T, Cavaco-Paulo A, Olsson L, Gatenholm P. Lipases efficiently stearate and cutinases acetylate the surface of arabinoxylan films. J Biotechnol. 2013;167:16-23.
11. Edgar KJ, Buchanan CM, Debenham JS, Rundquist PA, Seiler BD, Shelton MC, Tindall D. Advances in cellulose ester performance and application. Prog Polym Sci. 2001;26:1605-1688.

12. Heinze T, Liebert T. Unconventional methods in cellulose functionalization. Prog Polym Sci. 2001;26:1689-1762.

13. Song J, Rojas OJ. Approaching super-hydrophobicity from cellulosic materials: A Review. Nord Pulp Pap Res J. 2013;28: 216-238.

14. Daukantiene V, Bernotiene B, Gutauskas M. Textile hand: The influence of multiplex washing and chemical liquid softeners. Fibres Tex East Eur. 2005;13:63-66.

15. Tome LC, Freire MG, Rebelo LPN, Silvestre AJD, Neto CP, Marrucho IM, Freire CSR. Surface hydrophobization of bacterial and vegetable cellulose fibers using ionic liquids as solvent media and catalysts. Green Chem. 2011;13:2464-2470.

16. Garcia-Ubasart J, Colom JF, Vila C, Hernández NG, Blanca Roncero M, Vidal T. A new procedure for the hydrophobization of cellulose fibre using laccase and a hydrophobic phenolic compound. Bioresour Technol. 2012;112:341-344.

17. Chen S, Tong X, Woodard RW, Du G, Wu J, Chen J. Identification and characterization of bacterial cutinase. J Biol Chem. 2008;283:25854-25862.

18. Tzanov T, Calafell M, Guebitz GM, Cavaco-Paulo A. Biopreparation of cotton fabrics. Enzym Microb Technol. 2001;29: 357-362.

19. Gremos S, Zarafeta D, Kekos D, Kolisis F. Direct enzymatic acylation of cellulose pretreated in $\mathrm{BMIMCl}$ ionic liquid. Bioresour Technol. 2011;102:1378-1382.

20. Ghose TK. Measurement of cellulase activities. Pure Appl Chem. 1987;59:257-268.

21. Fu LN, Tan MY, Shi RK, Wang HB, Fu JJ. Insights into high temperature pretreatment on cellulase processing of bamboo. Therm Sci. 2012;16:1524-1528.

22. Fu JJ, Nyanhongo GS, Silva C, Cardinale M, Prasetyo EN, Yu CW, Cavaco-Paulo A, Gubitz GM. Bamboo fibre processing: Insights into hemicellulase and cellulase substrate accessibility. Biocatal Biotransfor. 2012;30:27-37.

23. Sereti V, Stamatis H, Pappas C, Polissiou M, Kolisis FN. Enzymatic acylation of hydroxypropyl cellulose in organic media and determination of ester formation by diffuse reflectance infrared Fourier transform (DRIFT) spectroscopy. Biotechnol Bioeng. 2001;72:495-500.

24. Das B, Das A, Kothari VK, Fangueiro R, Araújo M. Moisture transmission through textiles. Part I: Processes involved in moisture transmission and the factors at play. AUTEX Res J. 2007;7: 194-216.

25. Badenes S, Lemos F, Cabral JS. Transesterification of oil mixtures catalyzed by microencapsulated cutinase in reversed micelles. Biotechnol Lett. 2010;32:399-403.

26. Shahla S, Cheng N, Yusoff R. An overview on transesterification of natural oils and fats. Biotechnol Bioprocess Eng. 2010; 15:891-904

27. Badenes S, Lemos F, Cabral JS. Kinetics and mechanism of the cutinase-catalyzed transesterification of oils in AOT reversed micellar system. Bioprocess Biosyst Eng. 2011;34:1133-1142.

28. Khodaverdi M, Jeihanipour A, Karimi K, Taherzadeh M. Kinetic modeling of rapid enzymatic hydrolysis of crystalline cellulose after pretreatment by NMMO. $J$ Ind Microbiol Biotechnol. 2012;39:429-438.

Manuscript received Mar. 18, 2015, and revision received Oct. 22, 2015. 